\title{
The effect of processes measures of diabetes care and medication adherence on the developments of chronic diabetic complications
}

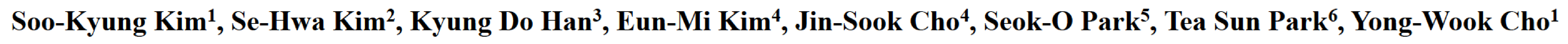

${ }^{1}$ Department of Internal Medicine, CHA Bundang Medical Center, CHA University, Seongnam, Korea; ${ }^{2}$ Department of Internal Medicine, Catholic Kwandong University, Incheon, Korea; ${ }^{3}$ Department of Biostatistics, College of Medicine, The Catholic University of Korea, Seoul, Korea; ${ }^{4}$ Health Insurance Review \& Assessment Service, Wonju, Korea;

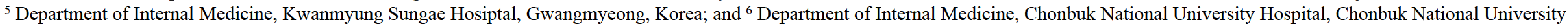
Medical School, Jeonju, Korea

\begin{abstract}
$\underline{\text { Abstract }}$
Background: Regular monitoring of glucose control and screening of diabetic complication and adherence to treatment are cornerstones for prevention of chronic diabetic complications. The aim of this study is to investigate the association between quality of diabetes care and the development of chronic diabetes complications.

Methods: This retrospective study was performed using the national health insurance claims database from the Health Insurance Review \& Assessment Service (HIRA) of Korea. Study patients were aged 30 years or older, had type 2 diabetes, had taking at least one hypoglycemic agent in only one attending clinic at 2009, and had no history of any diabetes-related chronic complications including cardiovascular diseases before December, 2010, cancer, or hospitalization for $\geq 90$ days $(\mathrm{n}=280,698)$. Patients who measured HbAlc and lipid profile at least once a year every year and had $\geq 80 \%$ of the possession rate of oral hypoglycemic medication every year from 2009 to 2014 were compared with those who didn't.

Results: The number of patients receiving 2 quality-of-care measures at least once a year increased to $52.5 \%$ in 2014 from $30.3 \%$ in 2009 . Those having $\geq 80 \%$ of the medication adherence also increased from $60.3 \%$ to $73.5 \%$. However, the patients who meet both conditions every year during study period were only $13,625(4.9 \%)$. In them, the cumulative incidence of microvascular complications (nephropathy and retinopathy) was $4.1 \%$, and that of macrovascular complications (myocardial and cerebral infarction and peripheral arterial disease) was $0.8 \%,(4.8 \%$ and $1.5 \%$, respectively in those who didn't).

Conclusions: The proportion of patients who received 2 quality-of-care measures and had $\geq 80 \%$ of the medication possession rate every year are seriously low, but they experienced fewer diabetic macrovascular complications. This result provides support for the importance for enhancing the quality of diabetes care.
\end{abstract}

Table 1. Percentage of patients receiving HIRA recommended care processes and state prescribing OHA by audit year

\begin{tabular}{|c|c|c|c|c|c|c|}
\hline & 2009 & 2010 & 2011 & 2012 & 2013 & 2014 \\
\hline $\mathrm{N}$ & 280,698 & 280,698 & 279,295 & 277,617 & 276,048 & 274,393 \\
\hline HbA1c measurement, more than once a year $(\mathrm{N},[\%])$ & $147,911(52.7)$ & $143,212(51.0)$ & $171,279(61.3)$ & $179,382(64.6)$ & $185,967(67.4)$ & $191,868(69.9)$ \\
\hline Lipid measurement, more than once a year $(\mathrm{N},[\%])$ & $91,329(32.5)$ & $91,722(32.7)$ & $110,723(39.6)$ & $119,631(43.1)$ & $126,988(46.0)$ & $149,417(54.5)$ \\
\hline $\operatorname{HbA1c}+\operatorname{Lipid}(\mathrm{N},[\%])^{*}$ & $84,119(30.0)$ & $85,482(30.5)$ & $105,227(37.7)$ & $114,411(41.2)$ & $122,350(44.3)$ & $144,110(52.5)$ \\
\hline Oral hypoglycemic agent possession ratio $\geq 80 \%(\mathrm{~N},[\%])$ & $169,146(60.3)$ & $188,736(67.2)$ & $194,501(69.6)$ & $199,322(71.8)$ & $202,437(73.3)$ & $201,626(73.5)$ \\
\hline Good quality of care $(\mathrm{N},[\%])^{* *}$ & $52,629(18.8)$ & $63,348(22.6)$ & $79,149(28.3)$ & $88,695(32.0)$ & $96,783(35.1)$ & $114,316(41.7)$ \\
\hline
\end{tabular}

${ }^{*}$ Measured at least once a year, respectively; ${ }^{* *} \mathrm{HbA} 1 \mathrm{c}$ measurement, at least once a year + lipid measurement, at least once a year $+\mathrm{OHA}$ possession ratio $\geq 80 \%$.

Table 2. Incidence of diabetic macrovascular and microvascular complications

\begin{tabular}{lcccc}
\hline & 2011 & 2012 & 2013 & 2014 \\
\hline Macrovascular complication (N, [\%]) & & & & \\
Myocardial infarct & $330(0.12)$ & $386(0.14)$ & $427(0.15)$ & $450(0.16)$ \\
Cerebral infarct & $535(0.19)$ & $516(0.19)$ & $585(0.21)$ & $646(0.24)$ \\
Peripheral arterial disease & $30(0.01)$ & $56(0.02)$ & $69(0.02)$ & $83(0.03)$ \\
All macrovascular complications & $890(0.32)$ & $948(0.34)$ & $1,073(0.39)$ & $1,166(0.42)$ \\
Microvascular complication (N, [\%]) & & & & \\
CKD, stage 3/4 & $203(0.07)$ & $510(0.18)$ & $817(0.30)$ & $1,066(0.39)$ \\
CKD, stage 5 & $481(0.17)$ & $927(0.33)$ & $1,343(0.49)$ & $1,775(0.65)$ \\
Replacement treatment & $35(0.01)$ & $98(0.04)$ & $195(0.07)$ & $343(0.13)$ \\
All CKD & $639(0.23)$ & $1,321(0.48)$ & $1,981(0.72)$ & $2,595(0.95)$ \\
Diabetic retinopathy & $1,015(0.36)$ & $1,601(0.58)$ & $2,088(0.76)$ & $2,580(0.94)$ \\
All microvascular complications & $1,620(0.58)$ & $2,828(1.02)$ & $3,932(1.42)$ & $4,967(1.81)$ \\
All chronic complication (N, [\%]) & $2,516(0.90)$ & $3,758(1.35)$ & $4,967(1.80)$ & $6,075(2.21)$
\end{tabular}

All microvascular complications

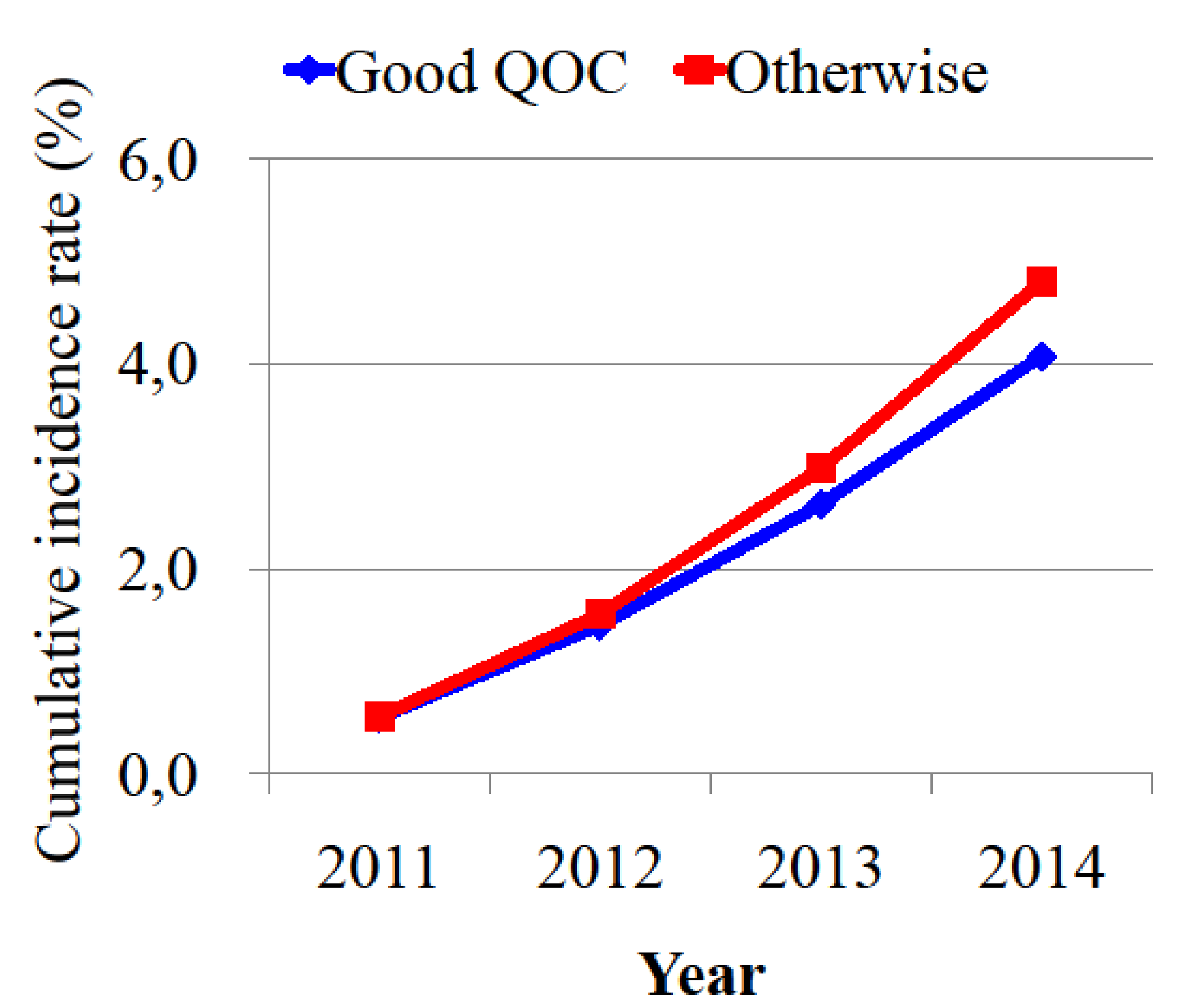

Figure 1. Cumulative incidence rate of diabetic complications between patients who had good quality of care (QOC) and those who didn't. A Good QOC was defined as measuring of HbA1c and lipid profile at least once a year every year and having $\geq$ $80 \%$ of the possession rate of oral hypoglycemic medication every year from 2009 to 2014.

All macrovascular complications

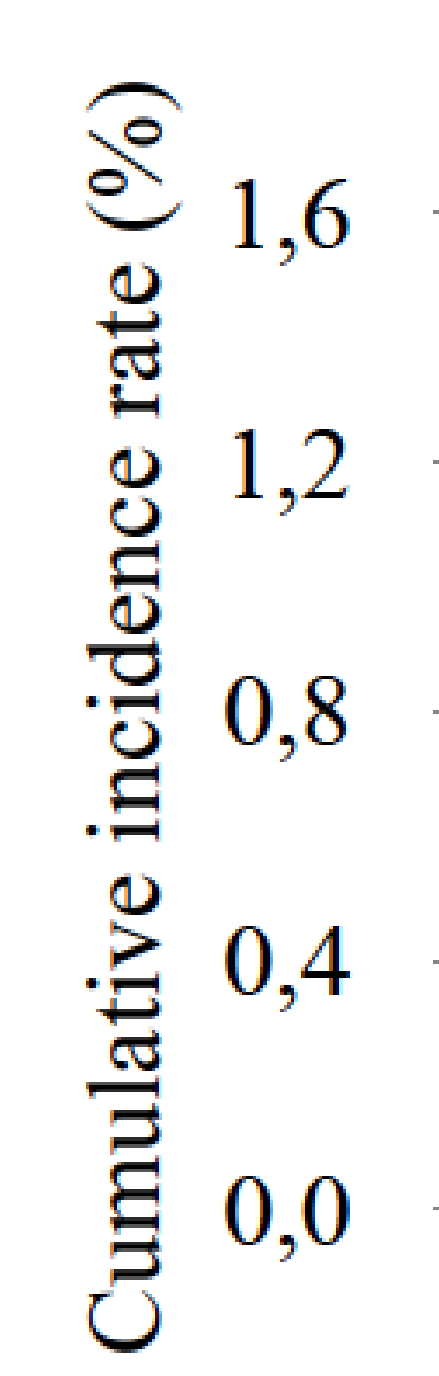

$\rightarrow$ Good QOC $=$ Otherwise

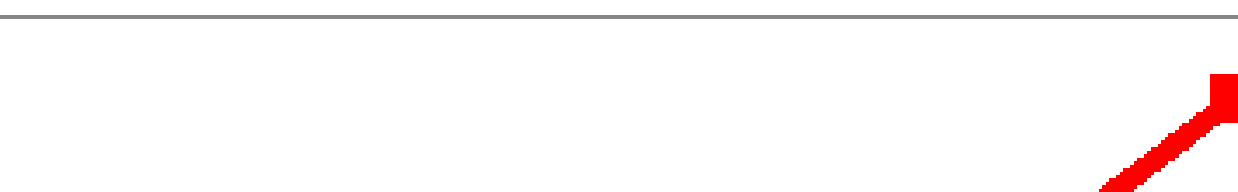

$20112012 \quad 2013 \quad 2014$

Yea 